\title{
Experimental Study of Magnetocaloric Effect in Dipolar Magnet $\mathrm{KEr}\left(\mathrm{MoO}_{4}\right)_{2}$
}

\author{
J. Hanko, M. Orendáč, M. KajŇaková, A. Orendáčová \\ AND A. FEHER
}

Centre of Low-Temperature Physics of Slovak Academy of Sciences and P.J. Šafárik University, Park Angelinum 9, 04154 Košice, Slovakia

We report on magnetothermodynamic properties of single crystal $\mathrm{KEr}\left(\mathrm{MoO}_{4}\right)_{2}$ which were investigated from nominally $0.4 \mathrm{~K}$ to $20 \mathrm{~K}$ in magnetic fields up to $4 \mathrm{~T}$. Using the available specific heat data the diagram of temperature and magnetic field dependence of the total entropy was constructed. Predictions for temperature dependences of the relative temperature variations during the adiabatic demagnetization and the entropy variation during the isothermal demagnetization were calculated from the specific heat data. The obtained results suggest that unlike common refrigerants, in $\mathrm{KEr}\left(\mathrm{MoO}_{4}\right)_{2}$ the quantities describing magnetic cooling remain nearly constant in the temperature range $2-10 \mathrm{~K}$.

PACS numbers: 75.30.Sg, 75.50.Ee

\section{Introduction}

Magnetocaloric effect (MCE) based on heating and cooling of matter in response to variations of external magnetic field has attracted much attention [1]. The independence of MCE of gravity makes it appropriate for space applications. Apart from the application potential the investigation of MCE gives insight in magnetic and thermodynamic properties of a studied system. Recently enhanced MCE was reported in low-dimensional and geometrically frustrated systems. In this work we present study of low-dimensional dipolar magnet $\operatorname{KEr}\left(\mathrm{MoO}_{4}\right)_{2}$. The material belongs to the class of low-dimensional dipolar magnets with strong anisotropy of easy axis type. The system is characterized by dipole interaction due to large magnetic moment of $\mathrm{Er}^{3+}$ ion $\left(13 \mu_{\mathrm{B}}\right)$ and their short distance $(3.957 \AA)$. Our previous study [2] identified the material as a representative of quasi-one-dimensional $S=3 / 2$ Blume-Capel model with single-ion anisotropy $D / k_{\mathrm{B}}=10.8 \mathrm{~K}$, intrachain coupling $J_{1} / k_{\mathrm{B}}=0.85 \mathrm{~K}$ and interchain coupling $J_{2} / k_{\mathrm{B}}=-0.15 \mathrm{~K}$.

\section{Experimental details}

The characteristic parameters describing magnetocaloric effect in $\mathrm{KEr}\left(\mathrm{MoO}_{4}\right)_{2}$ were calculated from specific heat data experimentally studied 
both in zero and non-zero magnetic field. Specifically, the experimental data below $2 \mathrm{~K}$ were studied using dual-slope method [3] in a ${ }^{3} \mathrm{He}-{ }^{4} \mathrm{He}$ dilution refrigerator from 0 to $130 \mathrm{mT}$. Specific heat data above $2 \mathrm{~K}$ were obtained in PPMS device in magnetic field varying from $0 \mathrm{~T}$ up to $4 \mathrm{~T}$. Magnetic field was created by superconducting magnet and oriented perpendicularly to $a c$ plane.

\section{Results and discussion}

Our previous experimental studies revealed that this system is characterized by a phase transition to the $\mathrm{AF}$ ordered state at about $T_{\mathrm{C}} \approx 0.95 \mathrm{~K}$ in zero magnetic field [2]. The position of the corresponding $\lambda$-like anomaly is shifted towards lower temperatures with increasing magnetic field. In addition, the width of the maximum is increased. It was found that the maximum in the heat capacity characterizing the phase transition is completely suppressed in magnetic fields higher than $100 \mathrm{mT}$. Specific heat data of $\mathrm{KEr}\left(\mathrm{MoO}_{4}\right)_{2}$ in zero magnetic field and magnetic field $4 \mathrm{~T}$ are presented in Fig. 1.

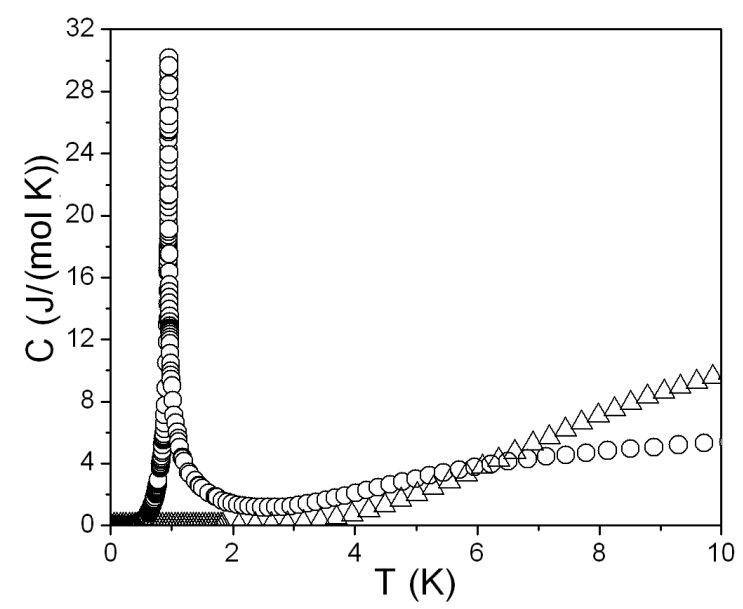

Fig. 1. Specific heat of $\mathrm{KEr}\left(\mathrm{MoO}_{4}\right)_{2}$ in zero magnetic field (empty circles) and magnetic field $4 \mathrm{~T}$ (empty triangles).

Temperature dependences of the total entropy for several values of magnetic field, namely, $0.75 \mathrm{~T}, 1 \mathrm{~T}, 2 \mathrm{~T}, 4 \mathrm{~T}$ were constructed from the specific heat data. The total entropy was calculated from the following equation: $S\left(T_{0}\right)=\int_{0}^{T_{0}} \frac{C(T)}{T} \mathrm{~d} T$ and the obtained data are presented in Fig. 2. Based on the observed specific heat behavior, using the equation $C(T) \sim T^{\alpha}$ seems to be reasonable for the extrapolation of specific heat data to zero temperature from $0.4 \mathrm{~K}$ and $2 \mathrm{~K}$ for zero magnetic field and fields higher than $0.7 \mathrm{~T}$, respectively.

Since the peak in specific heat data does not persist in magnetic fields higher than $100 \mathrm{mT}$ only the entropy in zero magnetic field possesses a sharp increase, in the vicinity of $1 \mathrm{~K}$. Notably, a crossover in the temperature dependence of 


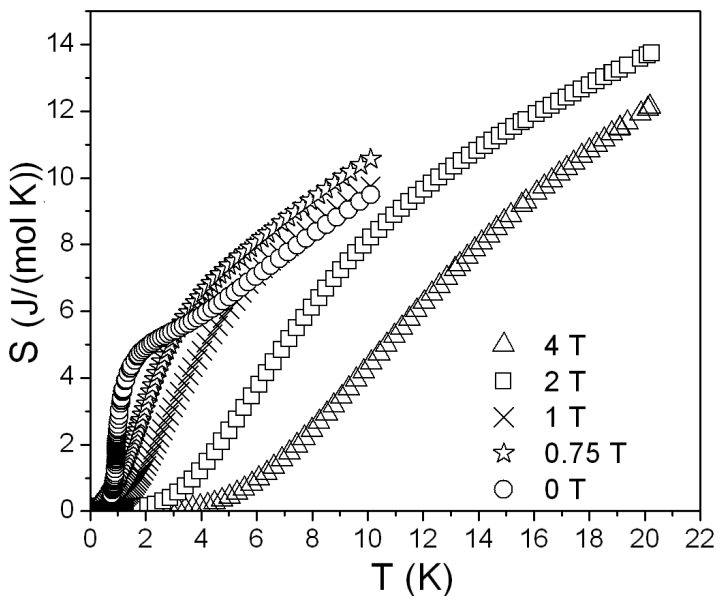

Fig. 2. Temperature dependence of total entropy calculated in various magnetic fields. See text for more detailed discussion.

the entropy calculated at $0 \mathrm{~T}$ and $0.75 \mathrm{~T}$ occurs, it can be attributed to the interplay between crystal field anisotropy, exchange coupling, Zeeman energy and temperature. Thanks to this crossover in the temperature dependence of entropy, anomalous MCE can be expected during demagnetization from $0.75 \mathrm{~T}$ to $0 \mathrm{~T}$ in temperatures above $3 \mathrm{~K}$ (see Fig. 2). The quantities characterizing the efficiency
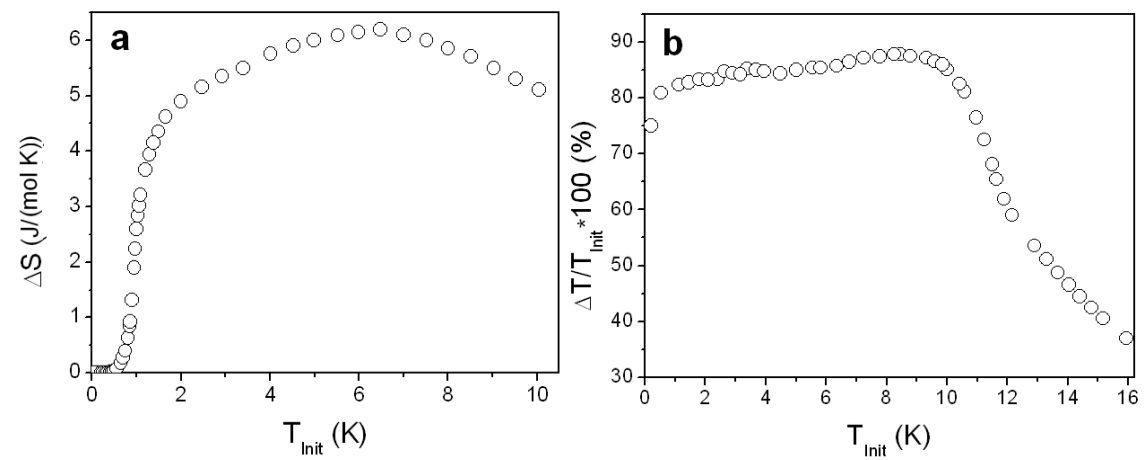

Fig. 3. Temperature dependence of total entropy change during isothermal demagnetization (a) and relative temperature change during adiabatic demagnetization (b) of $\operatorname{KEr}\left(\mathrm{MoO}_{4}\right)_{2}$ from $4 \mathrm{~T}$ to $0 \mathrm{~T}$.

of MCE namely entropy change during isothermal demagnetization and relative temperature change during adiabatic demagnetization were calculated from the entropy curves and for magnetic field change from $4 \mathrm{~T}$ to $0 \mathrm{~T}$ for different initial temperatures of the demagnetizations. The results of the calculations are illustrated in Fig. 3. The temperature dependence of the entropy change is characterized by a rapid increase near $1 \mathrm{~K}$ and broad maximum located near $6 \mathrm{~K}$. It could be noted 
that in relatively large temperature range from $2 \mathrm{~K}$ to $10 \mathrm{~K}$ the entropy change happens to be comparable with that of $S=1 / 2$ isotropic paramagnetic system $5.76 \mathrm{~J} /(\mathrm{mol} \mathrm{K})$, see Fig. 3a. Similarly, relative change of temperature manifests plateau up to $10 \mathrm{~K}$ (see Fig. 3b) followed by a rapid decrease. Unlike other types of compounds considered as refrigerants, in which single-ion anisotropy decreases the efficiency of magnetic cooling [4], crystal field in $\operatorname{KEr}\left(\mathrm{MoO}_{4}\right)_{2}$ flattens both characteristics in relatively wide temperature range. This unconventional behavior results from the energy level scheme, which is determined by the magnitude of characteristic parameters, namely, spin value, single-ion anisotropy, and exchange interactions on one side and Zeeman energy on the other side. Consequently, $\mathrm{KEr}\left(\mathrm{MoO}_{4}\right)_{2}$ should have the performance in magnetic cooling temperature independent of temperatures $2-10 \mathrm{~K}$ if demagnetized from $4 \mathrm{~T}$.

\section{Conclusion}

The total entropy, calculated from specific heat data studied from $0.4 \mathrm{~K}$ to nominally $20 \mathrm{~K}$ in magnetic fields up to $4 \mathrm{~T}$, revealed unconventional behavior above $3 \mathrm{~K}$ and at magnetic field $0.75 \mathrm{~T}$. The observed unusual dependence of the entropy change during isothermal demagnetization and relative temperature change during adiabatic demagnetization was attributed to the interplay between crystal field anisotropy, exchange coupling, Zeeman energy, and temperature. Further effort will be devoted to the direct experimental study of magnetocaloric effect in $\mathrm{KEr}\left(\mathrm{MoO}_{2}\right)_{4}$ in an extended range of parameters.

\section{Acknowledgments}

This work was supported by the Grant Agency VEGA — grant No. $1 / 3027 / 06$. The material support of U.S.Steel DZ-Energetika is gratefully acknowledged.

\section{References}

[1] A. Honecker, S. Wessel, Physica B 378-380, 1098 (2006) and references therein.

[2] A. Orendáčová, D. Horváth, M. Orendáč, E. Čižmár, M. Kačmár, V. Bondarenko, A. Anders, A. Feher, Phys. Rev. B 65, 014420 (2002).

[3] S. Riegel, G. Weber, J. Phys E, Sci Instrum. 19, 790 (1986).

[4] M. Evangelisti, A. Candini, A. Ghirri, M. Affronte, E.K. Brechin, E.J.L. McInnes, Appl. Phys. Lett. 87, 072504 (2005). 